\title{
Comparison of single versus multiple doses of antibiotic prophylaxis in reducing post-elective Caesarean section infectious morbidity
}

\author{
Shakya A, Sharma J \\ Tribhuvan University Teaching Hospital, Kathmandu, Nepal
}

\begin{abstract}
Background: Puerperal sepsis is frequently in Caesarean section. Antibiotic prophylaxis may have significant impact in reduction of infections and thus the need to study its role in sepsis prevention systematically.

Objective: The aim of this study is to compare the efficacy of single dose versus multiple doses of a first generation cephalosporin (with Metronidazole), to reduce postoperative infectious morbidity in elective caesarean section.

Materials and methods: It was prospective clinical trial of hundred women undergoing elective caesarean section who received either a single prophylactic dose of Cefazolin with Metronidazole post-cord clamping, or multiple postoperative doses of antibiotics based on the standard protocol of the hospital. Duration of the study was seven months and twentytwo days $\left(11^{\text {th }}\right.$ November 2004 to $30^{\text {th }}$ June 2005). Women were compared on the basis of development of postoperative febrile morbidity, endometritis, urinary tract infection, wound infection and other infections.

Results: There were no significant differences among the patients in single and multiple dose groups in terms of their age distribution, gravida, period of gestation, smoking status, body mass index, indications for elective caesarean section or operation characteristics. There were $4 \%$ and $6 \%$ febrile morbidity, $2(4 \%)$ and $0(0 \%)$ urinary tract infection, in the single dose and multiple dose groups respectively. But none of the differences were statistically significant.

Conclusion: A single prophylactic dose of Cefazolin plus Metronidazole given post-umbilical cord clamping gives as much protection as multiple postoperative doses of Cefazolin/Cefalexin plus Metronidazole in preventing postoperative infectious morbidity in elective caesarean section.
\end{abstract}

Key words: Antibiotic prophylaxis, Caesarean section

$\mathrm{C}$ aesarean section is a major cause of puerperal sepsis. The incidence of puerperal sepsis has been quoted as high as $36 \%$ compared with less than $1 \%$ for vaginal deliveries ${ }^{1}$. Puerperal sepsis is still a major cause of maternal mortality and morbidity. Puerperal morbidity may present in the form of a febrile morbidity, endometritis, cystitis and/or pyelonephritis, wound infection, mastitis, pelvic abscess, pulmonary atelectasis or septic pelvic thrombophlebitis.

Febrile morbidity, in the UK, is defined as a temperature of 38 degree Celsius or higher on any two of the first ten days postpartum except in the first 24 hours. In the USA, the threshold temperature is 37.8 degree Celsius. These numbers have been selected because they are convenient round numbers, rather than for any scientific reason. The first 24 hours have been excluded based on the high incidence of non-infective pyrexia especially if the patient is in labor ${ }^{1}$. To et $\mathrm{al}^{2}$ found that only the duration of labour over 10 hours was a significant risk factor for febrile morbidity. Suonio et $\mathrm{al}^{3}$ found postoperative hematoma, blood loss over $500 \mathrm{ml}$ and duration of labour exceeding 6 hours as risk factors for febrile morbidity.
Risk factors include caesarean section done after onset of labour, placement of open drains, obesity, and diabetes ${ }^{1}$. Careful operative technique to avoid hematoma formation, the avoidance of dead space during closure and intraoperative antibiotic prophylaxis are important preventive measures against wound infection.

Materials and methods

This study was conducted at Tribhuwan University Teaching Hospital, situated in Maharajgunj at Kathmandu valley in Nepal. It is a tertiary care centre, a referral hospital in Nepal. The settings for this study included the operation theatre, the post-operative ward, and the maternity ward in the department of Obstetrics at TUTH. The study was started on $11^{\text {th }}$ November 2004 and after a period of seven months and twenty two days, it was completed on $30^{\text {th }}$ June 2005 . Hundred

Correspondence

Dr. Abha Shakya

Tribhuvan University Teaching Hospital

Kathmandu, Nepal

E-mail: abhashakya@gmail.com 
women participated in this study among which 50 were in the study group and 50 in the comparative group. Purposive sampling technique was used as a sampling method. The study design was that of a prospective clinical trial. Patients undergoing elective caesarean section were included in the study. However, patients who were excluded from the study were those with known sensitivity to the medications used (Penicillin, cephalosporin and Metronidazole), alcohol consumption within previous 24 hours, liver disease, patients in labour, rupture of membranes, obvious infections, recent administration of antibiotics (within 7 days), diabetes mellitus, eclampsia/hypertension and anaemia.

\section{Results}

A total of 100 patients undergoing elective caesarean section, who met the inclusion and exclusion criteria of the study, were taken. Fifty patients were randomised to receive a single dose of prophylactic antibiotics (Cefazolin and Metronidazole) peroperatively whereas fifty received a standard seven-day course (multiple doses) of these antibiotics, continued postoperatively. There were 102 neonates delivered in these 100 patients. There was 1 set of twins each in the single and multiple dose groups. Number of patients with a previous scar of caesarean section was 13 and 14 in the single and multiple dose groups respectively.

The demographic data for women receiving a single dose of antibiotics were compared with those of the women receiving multiple doses (Table 1). Chi-Square test was used to measure the association between the age groups and the use of single and multiple doses of antibiotic prophylaxis among patients who underwent elective caesarean section. No significant differences were found $(\mathrm{P}=0.81)$. At the same time, in the same population, $\mathrm{Z}$ test was used to measure the mean age differences among single dose and multiple dose groups and no differences were found $(\mathrm{P}=0.98)$.

Qualitative variables like gravida and smoking status were also analysed using Chi-Square test and Fisher's Exact test in both single and multiple dose groups and no significant differences were observed ( $\mathrm{P}$ values 0.3 and 1 respectively). There were $34 \%$ primigravida and $66 \%$ multigravida in the single dose group, whereas $44 \%$ primigravida and $56 \%$ multigravida in the multiple dose group. There were 3 smokers $(6 \%)$ in the single dose group and 2 smokers $(4 \%)$ in the multiple dose groups. None of the smokers developed postoperative infectious morbidity.

Thus, the two groups of patients who received either single dose or multiple doses of prophylactic antibiotics were similar regarding age, gravida, gestational weeks, Body Mass Index (BMI) and smoking status.
The list of indications for elective caesarean section in women receiving single or multiple doses of prophylactic antibiotics is shown in Table 2. There were no important differences between the two groups. The commonest indications for elective caesarean section in both groups were previous scar of caesarean section, followed by cephalopelvic disproportion, breech presentation and bad obstetric history.

Caesarean section was done under spinal anaesthesia in most of the patients in the two groups (Single dose $94 \%$, multiple dose $90 \%$ ). Only $6 \%$ patients in the single dose group and $10 \%$ patients in the multiple dose group underwent caesarean section under general anaesthesia, due to failed spinal anaesthesia. No statistically significant differences were seen by the Fisher's Exact test $(\mathrm{P}=0.72)$.

All caesarean sections were done via Pfannenstiel incision. There were no complications during any of the operations. The duration of surgery was categorised based on the time taken as $<30$ minutes, 30 minutes to 1 hour and $>1$ hour. Operation lasted for $>1$ hour in $4 \%$ and $8 \%$ of women in the single and multiple dose groups respectively. Duration of operation was analyzed using Fisher's Exact test in relation to single and multiple doses of antibiotic prophylaxis used and no significant differences were found $(\mathrm{P}=0.13)$. The mean duration of operation \pm standard deviation in the single and multiple dose groups were $39.8+11.3$ minutes and $42.9+18.5$ minutes respectively and the $\mathrm{Z}$ test revealed no significant difference $(\mathrm{P}=0.31)$.

Majority of cases did not develop febrile morbidity. There were 6 cases of immediate postoperative infectious morbidity in these patients: 3 in the single dose group ( 1 febrile morbidity, 1 urinary tract infection, 1 febrile morbidity with urinary tract infection), and 3 in the multiple dose group ( 3 febrile morbidity). There was no febrile morbidity in $96 \%$ of patients and $94 \%$ of patients in the single dose and multiple dose groups respectively. Febrile morbidity in relation to single and multiple doses of antibiotic prophylaxis were analyzed using Fisher's Exact test and no statistically significant differences were observed $(\mathrm{P}=1)$.

Among the five patients who developed febrile morbidity, two patients (4\%) had received a single dose of prophylactic antibiotics. In one of these patients, two spikes of fever was seen 24 hours after operation, fever lasted for only one day, and it was not associated with other clinical features of infection. In the other patient, febrile morbidity was associated with urinary tract infection. 
On the other hand, three patients $(6 \%)$ developed febrile morbidity in the group that received multiple doses of antibiotics postoperatively. These women had two to three spikes of fever twenty-four to fortyeight hours after operation. Fever lasted for one day in two patients, and two days in one patient, and then subsided spontaneously. In all these patients, there were no associated clinical features of infection and their laboratory investigations revealed no evidence of infection.

Among the five women in both groups, who had febrile morbidity, only one woman had operative duration $>1$ hour (multiple dose group) whereas the rest had operations lasting less than an hour. The relation between duration of operation and febrile morbidity was further analyzed by Chi-Square test and it was significant in the single dose group $(\mathrm{P}=0.003)$ and not significant in the multiple dose group $(\mathrm{P}=0.22)$.

Both women, who had febrile morbidity in the single dose group, had undergone operation under GA whereas women who developed the same in the multiple dose groups had received spinal anaesthesia. The relation between mode of anaesthesia (GA vs. spinal) and dose of antibiotics (single vs. multiple) was analyzed using Chi-Square test and it was not statistically significant (single dose, $\mathrm{P}=0.72$; multiple dose, $\mathrm{P}=0.17$ ).

Between the two women who developed febrile morbidity in the single dose group, one was obese (BMI>30). Among the three women who had febrile morbidity in the multiple dose groups, two were obese. However the relation between BMI and febrile morbidity was not statistically significant as assessed by the Chi-Square test (single dose, $\mathrm{P}=0.34$; multiple dose, $\mathrm{P}=0.59$ ).

There were also no significant differences among the indications for caesarean section in women who developed febrile morbidity in single and multiple dose groups (single dose, $\mathrm{P}=0.99$; multiple dose, $\mathrm{P}=0.10$ ).

There were no cases of urinary tract infection in the group that received multiple doses of antibiotics. Two patients $(4 \%)$ who received single prophylactic dose of antibiotics developed urinary tract infection whereas
$96 \%$ did not. But the $\mathrm{P}$ value is 0.50 as calculated by Fisher's Exact test, indicating that this difference is also not statistically significant.

Between the two patients who developed urinary tract infection, one had associated febrile morbidity while the other was afebrile. One patient had been catheterised continuously for three postoperative days due to haematuria resulting from slight iatrogenic injury to the urinary bladder during the use of diathermy. She had normal preoperative urine microscopy. But once her catheter was removed after 72 hours of operation, she developed high grade fever associated with dysuria. Her urine microscopy showed plenty of pus cells. Urine was sent for culture and oral Ampicillin 500mg 6 hourly was started without waiting for the culture results. Later urine culture showed the growth of Escherechia coli after 48 hours, sensitive to Ampicillin. The patient was prescribed Ampicillin for total ten days duration.

The other patient who developed urinary tract infection had been catheterised for twenty-four hours postoperatively, which is the usual practice in cases of caesarean section at TUTH. Her preoperative urine microscopy examination was also normal. She developed dysuria on the third postoperative day, not associated with fever. Her urine microscopy revealed 8-10 pus cells per high power field. So she was given oral Amoxycillin 500mg 8 hourly for ten days. Urine culture report was available after 48 hours, which failed to grow any organisms.

Both women with UTI in the single dose group had operative duration less than an hour. Both of them had caesarean section under spinal anaesthesia. One woman was obese and the other had normal BMI. No statistically significant relation was found among mode of anaesthesia, BMI, duration of operation, indications for caesarean section with urinary tract infection in single and multiple dose groups, by Chi-Square test $(\mathrm{P}=0.72, \mathrm{P}=0.34, \mathrm{P}=0.91$ and $\mathrm{P}=0.98$ respectively $)$.

There were no cases of endometritis, wound infection or other infections in either single dose or multiple dose groups. No serious adverse reactions or intolerance was reported with either antibiotic regimen. 
Table 1: Epidemiological and obstetric characteristics of the patients in single and multiple dose groups

\begin{tabular}{|c|c|c|c|c|c|}
\hline & \multicolumn{2}{|c|}{ Single dose $(n=50)$} & \multicolumn{2}{|c|}{ Multiple dose $(n=50)$} & \multirow[t]{2}{*}{ P value } \\
\hline & Number & Percent & Number & Percent & \\
\hline Age (Years) & & & & & \\
\hline$<20$ & 2 & 4 & 2 & 4 & \\
\hline $20-29$ & 34 & 68 & 31 & 62 & 0.81 \\
\hline$>29$ & 15 & 28 & 17 & 34 & \\
\hline Mean age $\pm \mathrm{SD}$ & \multicolumn{2}{|c|}{$26.8 \pm 5.2$} & \multicolumn{2}{|c|}{$26.8 \pm 5.0$} & 0.98 \\
\hline $\begin{array}{l}\text { Gestation (weeks): } \\
\text { Mean GA } \pm \mathrm{SD}^{*}\end{array}$ & \multicolumn{2}{|c|}{$38.03 \pm 0.9$} & \multicolumn{2}{|c|}{$38.3 \pm 1.2$} & 0.82 \\
\hline \multicolumn{6}{|l|}{ Gravida: } \\
\hline Primi & 17 & 34.0 & 22 & 44.0 & 0.31 \\
\hline Multi & 33 & 66.0 & 28 & 56.0 & \\
\hline \multicolumn{6}{|l|}{ Body mass index: } \\
\hline Thin $(<20)$ & 0 & 0 & 3 & 6.0 & 0.14 \\
\hline Normal (20-24) & 14 & 28.0 & 11 & 22.0 & \\
\hline Mild overweight (24-30) & 25 & 50.0 & 19 & 38.0 & \\
\hline Obese $(>30$ & 11 & 22.0 & 17 & 34.0 & \\
\hline Mean $\mathrm{BMI} \pm \mathrm{SD}^{\#}$ & \multicolumn{2}{|c|}{$27.4 \pm 4.9$} & \multicolumn{2}{|c|}{$28 \pm 4.3$} & 0.52 \\
\hline \multicolumn{6}{|l|}{ Smoker: } \\
\hline & & & & & 1.0 \\
\hline Yes & 3 & 6.0 & 2 & 4.0 & \\
\hline No & 47 & 94.0 & 48 & 96.0 & \\
\hline
\end{tabular}

* $\mathrm{GA}=$ gestational age, $\mathrm{SD}=$ standard deviation ${ }^{*} \mathrm{BMI}=$ body mass index

Table 2: Indications for elective caesarean section in single and multiple dose groups.

\begin{tabular}{|c|c|c|c|c|}
\hline \multirow{3}{*}{$\begin{array}{l}\text { Indications for elective } \\
\text { cesarean section }\end{array}$} & \multicolumn{4}{|c|}{ Antibiotic regime administered: } \\
\hline & \multicolumn{2}{|c|}{ Single Dose $(n=50)$} & \multicolumn{2}{|c|}{ Multiple Dose $(n=50)$} \\
\hline & Number & Percent (\%) & Number & Percent(\%) \\
\hline Previous caesarean & 18 & 36.0 & 16 & 32.0 \\
\hline CPD* & 10 & 20.0 & 10 & 20.0 \\
\hline Bad Obstetric History & 6 & 12.0 & 5 & 10.0 \\
\hline Breech presentation & 9 & 18.0 & 8 & 16.0 \\
\hline Oblique lie & 2 & 4.0 & 0 & 0.0 \\
\hline Primary subfertility & 2 & 4.0 & 2 & 4.0 \\
\hline $\mathrm{IUGR}^{\#}$ & 1 & 2.0 & 1 & 2.0 \\
\hline Placenta praevia & 1 & 2.0 & 1 & 2.0 \\
\hline Twins & 1 & 2.0 & 1 & 2.0 \\
\hline Transverse lie & 0 & 0.0 & 3 & 6.0 \\
\hline Rectovaginal fistula & 0 & 0.0 & 1 & 2.0 \\
\hline $\begin{array}{l}\text { Cord around the neck on } \\
\text { Ultrasound }\end{array}$ & 0 & 0.0 & 1 & 2.0 \\
\hline Right hip joint dislocation & 0 & 0.0 & 1 & 2.0 \\
\hline
\end{tabular}

* $\mathrm{CPD}=$ cephalopelvic disproportion "IUGR=intrauterine growth ceiling

\section{Discussion}

Performing caesarean section increases a woman's risk of infectious morbidity. This risk is reduced by: a/ following recommended infection prevention practices, $\mathrm{b} /$ providing prophylactic antibiotics at the time of the procedure. Prophylactic antibiotics are given to help prevent infection. Only if a woman is suspected to have or is diagnosed as having an infection, therapeutic antibiotics is more appropriate ${ }^{4}$.

WHO recommends giving prophylactic antibiotics at caesarean section, when the umbilical cord is clamped after delivery of the baby. One dose of prophylactic 
antibiotics is sufficient and is no less effective than 3 doses or 24 hours of antibiotics in preventing infection. If the procedure lasts longer than 6 hours or blood loss is $1500 \mathrm{ml}$ or more, a second dose of prophylactic antibiotic is recommended to maintain adequate blood levels during the procedure ${ }^{4}$.

The use of perioperative antibiotics is a well established practice. The Cochrane collaboration published a systematic review, examining the efficacy of antibiotic prophylaxis in preventing infections after a caesarean section ${ }^{5}$. One review examined the efficacy of prophylaxis in elective and non-elective procedures, and concluded that prophylaxis has a strong protective effect for all types of caesarean section ${ }^{5}$. The other review examined specific prophylactic regimens, and concluded that Cefazolin and Ampicillin, administered as a single dose, were equally efficacious ${ }^{6}$. Broader spectrum antibiotics, and administration of more than one dose were not more efficacious.

At Tribhuwan University Teaching Hospital in Nepal, since the early 1990s, the combination of intravenous Cefazolin $500 \mathrm{mg} 6$ hourly plus Metronidazole $2 \mathrm{gm}$ intravenous single dose, followed by oral Cefalexin has been used postoperatively for both elective and non-elective caesarean sections. This regime has been expected to give coverage against gram-positive, gramnegative bacteria including anaerobes. These drugs are comparatively cheaper than the broad/extended spectrum antibiotics. Cefazolin plus Metronidazole was found to be superior to Cefazolin alone for antibiotic prophylaxis at caesarean section ${ }^{7}$ due to a reduction in postoperative infectious morbidity.

In this study, the most common postoperative infectious complication was found to be febrile morbidity. Two and three patients (4\% and $6 \%$ ) developed febrile morbidity in the group which received single dose antibiotics intraoperatively and the group which received postoperative multiple dose regime respectively. This difference was not statistically significant, which is comparable to the studies done by Rouzi et $\mathrm{al}^{8}$, Dimitrov et $\mathrm{al}^{9}$, Jacobi et $\mathrm{al}^{10}$ and Bagratee et $\mathrm{al}^{11}$. Only one patient who developed febrile morbidity in the single dose group of this study, had associated cause of infectious morbidity as urinary tract infection. In the rest of the patients, in both single and multiple dose groups, there were no associated clinical features of infection nor were there any laboratory evidence of infection. Their first spike occurred within twenty-four hours of operation; temperature remained elevated till twenty four to fortyeight hours after surgery, and returned to normal within forty-eight hours of surgery.

Two women in the single dose group developed urinary tract infection. One woman developed UTI after being catheterised for 72 hours postoperatively due to minor iatrogenic injury to the urinary bladder during operation. The other woman developed afebrile dysuria on the third postpartum day. The preoperative urine microscopy results of both patients were normal. There were no statistically significant differences in the mode of anaesthesia, BMI, duration of operation and indications of caesarean section in the women with UTI $(\mathrm{P}=0.72, \mathrm{P}=0.34, \mathrm{P}=0.91$ and $\mathrm{P}=0.98$ respectively).

Occult bacteriuria, bladder trauma, and catheterisation are risk factors for UTI. ${ }^{1}$ Moreover, proper aseptic precautions during insertion of catheter are also important to prevent infection. While some may doubt the protective coverage of single dose of prophylactic antibiotic against urinary tract infection especially in cases with prolonged catheterisation, note that $96 \%$ of patients with similar epidemiological / surgical characteristics did not develop infection with the same regimen. Moreover, the difference in the rate of UTI in single and multiple dose groups is not statistically significant $(\mathrm{P}=0.50)$.

There were no cases of endometritis in this study. Protection against endometritis is corroborated by other studies 5 . Risk factors for endometritis include caesarean section, chorioamnionitis, prolonged rupture of membranes, premature labour, multiple vaginal examinations, retained products of conception and low socioeconomic status ${ }^{1}$. The absence of endometritis in both single and multiple dose groups, therefore, maybe because women having elective caesarean section with intact membranes do not have sufficient inocculum of cervico-vaginal organisms for colonisation of endometrium. On the other hand, it also indicates that a single dose of Cefazolin plus Metronidazole prophylaxis rather than a prolonged course, maybe adequate to prevent endometritis.

There were also no cases of wound infection in this study. Neither single nor multiple dose regimens altered the wound infection rate. Risk factors for wound infection include duration of operation over 1 hour, onset of labor, ruptured membranes, placement of open drains, obesity, diabetes, puerperal endometritis and vertical skin incisions ${ }^{1,3}$. A high preoperative haematocrit is also protective $^{12}$.

There were $11(22 \%)$ and $17(34 \%)$ patients in the single and multiple dosage group with BMI belonging to the "obese" category, based on Naeye's classification for BMI of pregnant women ${ }^{13}$. None of these patients developed wound infection in either group, which may suggest that a single dose of Cefazolin plus Metronidazole prophylaxis was as protective against wound infection, irrespective of BMI. However, 
such an inference should be based on a larger sample size. Also, none of the patients were in labour, nor had ruptured membranes, blood loss $>500 \mathrm{ml}$ or any intraoperative complications. All women had $>10 \mathrm{gm} /$ dl pre- and postoperative haemoglobin and none had a vertical skin incision during caesarean section. These parameters could also have influenced the presence of wound infection. Moreover, absence of wound infection is also determined by careful operative technique to avoid hematoma formation, the avoidance of dead space during closure, intra and postoperative wound care principles.

Two women with febrile morbidity in the single dose group and one (out of three) woman in the multiple dose group were operated under general anaesthesia. But the relation between mode of anaesthesia (GA vs. spinal) and dose of antibiotics was not significant $(\mathrm{P}>0.05)$.

A small sample size is one limitation of this study. This prevents generalising the findings of this study on to the rest of the population. Another limitation of this study is that it aims to study only the immediate postoperative infectious morbidity, and not the long term protective effects of antibiotics against infection. Though all these patients were followed up postpartum when they came to the outpatient department for stitch removal on the seventh or tenth postpartum day, and again at 30 days postpartum, and none of these patients had any infectious complication requiring treatment or admission, we do not know of the events in the interim period when these women didn't have to come for follow-up.

A control group receiving no antibiotics would have been useful to reduce bias in this study, but it couldn't be incorporated due to ethical reasons. Unpredictable infection prevention practices in our set-up were the main reason for this.

This study did not address the cost-analysis of Cefazolin and Metronidazole and their potential for emergence of resistant organisms. Both are vast areas, not within the scope of this small clinical trial.

\section{Conclusion}

Since the differences in the rates of febrile morbidity and urinary tract infection were not statistically significant, and there were no wound infection, endometritis or other infections in women who received either single dose or multiple doses of prophylactic antibiotics for elective caesarean section in this study, it may be argued that both single and multiple dose regimen protected equally against post-elective caesarean section infectious morbidity and that a single prophylactic dose prevents unnecessary long course of antibiotics and susceptibility to antibiotic resistance.
Though the objective of this study did not include costeffectiveness of either regime, the cost of antibiotic therapy is apparently reduced in the single dose group. However, prophylactic antibiotics should not replace proper pre- and intra operative preparation and meticulous surgical technique.

\section{References}

1. Steer JP. Puerperal sepsis. In: Chamberlain G, Steer P, editors. Turnbull's Obstetrics. 3rd ed. London: Churchill Livingstone;2001.p.663-70.

2. To WW, Lau WN. A protocol of selective antibiotic prophylaxis for caesarean section based on risk factors. Aust N Z J Obstet Gynaecol. 2001;41:402-6.

3. Suonio S, Saarikoski S, Vohlonen I, Kauhanen O. Risk factors for fever, endometritis and wound infection after abdominal delivery. Int J Gynaecol Obstet. 1989;29:135-42.

4. World Health Organisation. Managing complications in pregnancy and childbirth: a guide for midwives and doctors. 2002:C35-6.

5. Smaill F, Hofmeyr GJ. Antibiotic prophylaxis for cesarean section. Cochrane Database Syst Rev. 2002;3:CD000933.

6. Hopkins L, Smaill F. Antibiotic prophylaxis regimens and drugs for cesarean section. Cochrane Database Syst Rev. 2000;2: CD001136.

7. Meyer NL, Hosier KV, Scott K, Lipscomb GH. Cefazolin versus cefazolin plus metronidazole for antibiotic prophylaxis at cesarean section. South Med J. 2003;96:992-5.

8. Rouzi AA, Khalifa F, Ba'aqueel H, Al-Hamdan HS, Bondagji N. The routine use of cefazolin in cesarean section.Int J Obstet Gynecol. 2000;69:107-12.

9. Dimitrov A, Pŭnevska M, Dikov I, Nikolov A, Kostov I. Prophylaxis with tercef of infectionrelated complications after cesarean section. Akush Ginekol. 2001;42:33-7.

10. Jakobi P, Weissman A, Zimmer EZ, Paldi E. Single-dose cefazolin prophylaxis for cesarean section. Am J Obstet Gynecol. 1988;158:1049-52.

11. Bagratee JS, Moodley J, Kleinschmidt I, Zawilski W. A randomised controlled trial of antibiotic prophylaxis in elective caesarean delivery. BJOG. 2001;108:143-8.

12. Wall PD, Deucy EE, Glantz JC, Pressman EK. Vertical skin incisions and wound complications in the obese parturient. Obstet Gynecol. 2003; 102(5Pt 1):952-6.

13. Dickinson JE. Cesarean section. In: James DK, Steer PJ, Weiner CP, Gonik B, editors. Highrisk pregnancy management options. 2nd ed. London: Harcourt Publishers; 1999.p.1217-29. 